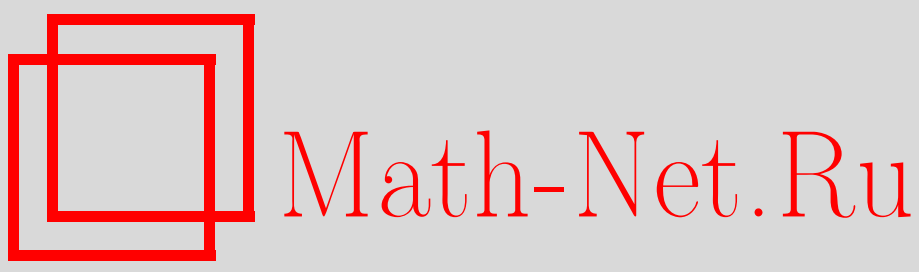

Б. Ю. Стернин, В. Е. Шаталов, Б.-В. Шульц, Об общих краевых задачах для эллиптических уравнений, Матем. сб., 1998, том 189, номер 10, 145-159

DOI: https://doi.org/10.4213/sm357

Использование Общероссийского математического портала Math-Net.Ru подразумевает, что вы прочитали и согласны с пользовательским соглашением http://www . mathnet.ru/rus/agreement

Параметры загрузки:

IP : 54.197 .217 .227

26 апреля 2023 г., $14: 35: 51$ 


\section{Об общих краевых задачах для эллиптических уравнений}

\begin{abstract}
Строится теория общих краевых задач для эллиптических дифференциальных операторов, символы которых не обязательно удовлетворяют условиям Атья-Ботта обращения в нуль соответствующего препятствия. Вводится условие эллиптичности краевой задачи и доказывается соответствующая теорема конечности (фредгольмовости).

Библиография: 29 названий.
\end{abstract}

\section{Введение}

Теория краевых задач для эллиптических дифференциальных уравнений в пространствах Соболева в настоящее время хорошо известна (см., например, [1], [2]). Основная теорема, касающаяся этих задач, состоит в том, что при некоторых алгебраических условиях (условиях Шапиро-Лопатинского) соответствующие операторы являются фредгольмовыми. При этом одной из важных характерных черт этой теории является то, что не любой эллиптический оператор на многообразии с краем допускает краевые условия указанного выше типа. Более того, было найдено (см. [3]), что препятствие к существованию (псевдодифференциальных) фредгольмовых задач в пространствах Соболева имеет топологический характер, и поэтому данный эллиптический оператор допускает краевую задачу типа Фредгольма тогда и только тогда, когда соответствующее препятствие исчезает [4], [5].

$\mathrm{K}$ сожалению, это препятствие не исчезает для некоторых важных геометрических операторов, таких, например, как оператор Хирцебруха (сигнатуры) или оператор Дирака. Отсюда, в частности, следует, что обшая формула для индекса эллиптических операторов для многообразия с краем (см., например, [4], [6]) не может быть применена к этим операторам.

Попытка найти формулу для сигнатуры в случае многообразия с краем привела Атью-Патоди-Зингера [7] к рассмотрению краевых задач для оператора Хирцебруха (и Дирака) в пространстве $L_{2}$. Более точно, эти операторы представляются как неограниченные операторы в пространстве $L_{2}$ с областью определения, связанной с однородньми краевьми условиями специального вида. В этой ситуации упомянутые выше операторы являются фредгольмовыми и, например, индекс оператора Хирцебруха на многообразии с краем выражается в терминах $L$-рода, т.е.

Работа вьполнена при финансовой поддержке Deutsche Forschungsgemeinschaft и Российского фонона фундаментальных исследований (гранты № 97-01-00703 и № 97-02-16722а).

(С) Б. Ю. Стернин, В. Е. ШАТАЛОв, Б.-В. ШуЛЬце 1998 
интеграла от полинома Хирцебруха от классов Понтрягина [8] и некоторого добавочного члена, назьваемого $\eta$-инвариантом [7]. Итак, возникают два совершенно различных случая: в то время как для эллиптического дифференциального оператора с краевыми условиями типа Шапиро-Лопатинского можно рассматривать общую краевую задачу саму по себе или рассматривать этот же оператор как неограниченный оператор в $L_{2}$, соответствуюший однородным граничным условиям, для операторов, не допускающих условия Шапиро-Лопатинского, имеется лишш вторая возможность. В связи с этим возникает следуюший вопрос: возможно ли построить фредгольмову теорию общих неоднородных краевых задач для произвольных әллиптических операторов (которая, разумеется, включала бы классические операторы, т.е. удовлетворяющие условию ШІапиро-Лопатинского)? В частности, такая теория должна включать в себя и неоднородные краевые задачи для оператора Хирцебруха и Дирака. В настояшей статье мы и предлагаем такую теорию.

Сначала кратко сформулируем основную идею, которая была использована в данной работе. Простые примеры для уравнения Коши-Римана и Бицадзе [9], а также и для других уравнений подобного типа показывают, что хотя они и не обладают свойством фредгольмовости в пространствах Соболева, однако корректные (фредгольмовы) краевые задачи могут быть поставлены, если правые части в краевых условиях принадлежат более тонкому пространству, чем пространство Соболева. Например, для уравнений Коши-Римана - это пространство типа Харди [10], [11]). В действительности, эти пространства суть (замкнутые) подпространства некоторых пространств Соболева, что позволяет высказать предположение, что в общем случае нужно брать правые части в краевых условиях не из всего пространства Соболева, а из некоторого его подпространства. Эта идея и является ключевой. Более точно, рассматривается $(m-1)$-й джет решений однородного дифференциального уравнения порядка $m$, взятый в точке границы. Это пространство является подпространством соболевского пространства сечений соответствуюшего расслоения над краем, что дает тривиальньй пример краевой задачи для произвольного эллиптического оператора. В классической теории краевых задач граничньй оператор может быть рассмотрен как изоморфное (или почти изоморфное, т.е. фредгольмово) отображение этого подпространства на соболевское пространство сечений некоторого другого расслоения многообразия над краем. В неклассической (общей) теории краевых задач коранг такого рода отображения может быть бесконечномерным. С топологической точки зрения препятствие к существованию классической краевой задачи эквивалентно несуществованию изоморфизма некоторого векторного расслоения над $T_{0}^{*} X=T^{*} X \backslash\{0\}$, где $X$ - край, обратному образу некоторого расслоения над $X$. С аналитической точки зрения препятствие состоит в несушествовании псевдодифференциального почти изоморфизма меж ду определенным подпространством соболевского пространства джетов на границе и пространством Соболева сечений векторного расслоения над $X$. Легко проверить, что последнее условие является "квантованием" первой (топологической) версии.

Статья состоит из двух параграфов. Первый параграф содержит основные результаты. Именно, в п. 1.1 вводится и обсуждается понятие общих краевых задач; в п. 1.2 дается критерий фредгольмовости; в п. 1.3 описывается псевдодифферен- 
циальная постановка общих краевых задач и доказьвается соответствующая теорема конечности. Наконец, в п. 1.4 обсуждается, что означают в этом контексте условия Шапиро-Лопатинского.

В $\S 1$ сушественно используются так называемые проекторы Кальдерона-Сили (часто называемые проекторами Кальдерона) [12]-[14]. Их конструкция включает оператор, обратньй к эллиптическому оператору, определяемому на дубле начального многообразия. Использование такого рода оператора несколько неудобно, поскольку интуитивно ясно, что все, что касается краевых условий, должно в большей степени определяться поведением оператора вблизи края (или даже на крае!), чем на всем многообразии (не говоря уже о проблеме продолжения оператора на дубль). Это обстоятельство явилось причиной, по которой мы предпочли доказательство теоремы конечности с помощью явного построения параметрикса.

Параграфф 2 содержит два простых и известных, но важных в геометрическом отношении примера, один из которых не допускает классических краевых задач (оператор Коши-Римана, п. 2.1), в то время как другой (оператор Эйлера, п. 2.2), как известно, обладает классическими краевыми задачами.

Предварительная версия этой работы вышла в 1997 году в Германии [15]. Она была рассказана на конференции по дифференциальным уравнениям в Потсдаме (осень 1997 года), а также на семинарах профессора А.С. Мищенко и профессора А. Т. Фоменко в Московском государственном университете (зима 1998 года).

Авторы выражают благодарность В.Е. Назайкинскому, за внимательное прочтение настоящего текста и за сделанные им полезные замечания и улучшения.

\section{§1. Основные результаты}

1.1. Определение общих краевых задач. Пусть $M-$ компактное гладкое многообразие с гладкой границей $\partial M=X$ и пусть

$$
\widehat{D}: C^{\infty}(M, E) \rightarrow C^{\infty}(M, F)
$$

где $E$ и $F$ - векторные расслоения над $M, V$ - эллиптический дифференциальньй оператор порядка $m$ на $M$. Мы дадим абстрактное понятие общих краевых задач (BVP) для оператора (1) (которое включает классические краевые задачи в качестве специального случая), введем специальную конструкцию общих краевых задач и покажем, что эта конструкция всегда определяет нам фредгольмову краевую задачу в подходящих функциональных пространствах. В качестве побочного результата мы получим хорошо известное условие сушествования классических краевых задач - условие Шапиро-Лопатинского.

Как обычно, в эллиптической теории оператор (1) рассматривается в соболевских пространствах

$$
\widehat{D}: H^{s}(M, E) \rightarrow H^{s-m}(M, F)
$$

Здесь для простоты число $s$ предполагается цельм. Краевые условия могут быть наложены на $(m-1)$-й джет $j_{X}^{m-1}(u)$ решения $u \in H^{s}(M, E)$ в точках границы. 
При этом предполагается, что воротниковая окрестность $U$ края $X$ в $M$ отождествляется с произведением $X \times[0,1)$ (это может быть сделано, например, посредством введения римановой метрики на $M$, при этом $(x, t) \in X \times[0,1)$ идентифицируется с точкой, расположенной на расстоянии $t$ от $X$ вдоль геодезической, исходящей из $x \in X$ в направлении внутренней нормали). По теореме о следах мы имеем непрерывное отображение

$$
j_{X}^{m-1}: H^{s}(M, E) \rightarrow \mathscr{H}_{m}^{s-1 / 2}(X, E) \equiv \bigoplus_{j=0}^{m-1} H^{s-1 / 2-j}\left(X, i^{*} E\right),
$$

которое сопоставляет каждому сечению $u \in H^{s}(M, E)$ джет $(m-1)$-го порядка

$$
j_{X}^{m-1}(u)=\left(\left.u\right|_{X},-\left.i \frac{\partial u}{\partial t}\right|_{X}, \ldots,\left.\left(\left(-i \frac{\partial}{\partial t}\right)^{m} u\right)\right|_{X}\right)
$$

в точках края $\left(i^{*} E-\right.$ обратный образ расслоения $E$ при вложении $i: X \hookrightarrow M$, ограничение $\left.\varphi\right|_{X}$ определяется следующим образом:

$$
\left.\varphi\right|_{X}=\left.\lim _{\tau \rightarrow 0+} \varphi\right|_{t=\tau}
$$

причем предел берется в соответствуюшем соболевском пространстве на $X$ ). Далее для краткости будем писать $E$ вместо $i^{*} E$.

ОПРЕДЕЛЕНИЕ 1. Общей краевой задачей оператора (2) мы называем задачу вида

$$
\left\{\begin{array}{l}
\widehat{D} u=f \in H^{s-m}(M, F), \\
\widehat{B}\left(j_{X}^{m-1}(u)\right)=g \in \mathscr{L}
\end{array}\right.
$$

для неизвестной функции $u \in H^{s}(M, E)$, где $\mathscr{L}$ - банахово пространство, а

$$
\widehat{B}: \mathscr{H}_{m}^{s-1 / 2}(X, E) \rightarrow \mathscr{L}
$$

- непрерывный линейный оператор.

Другими словами, общая краевая задача есть оператор вида

$$
\left(\widehat{D}, \widehat{B} \circ j_{X}^{m-1}\right): H^{s}(M, E) \rightarrow H^{s-m}(X, E) \oplus \mathscr{L},
$$

где $\widehat{D}$ и $\widehat{B}$ - операторы вида (2) и (4).

ЗАмЕчАниЕ 1. Классическая краевая задача является специальньм случаем задачи (3), в котором $\mathscr{L}$ - пространство Соболева сечений некоторого расслоения $X$, а $\widehat{B}$-псевдодифференциальный оператор.

Основной причиной для введения понятия обших BVP является то, что для данного оператора $\widehat{D}$ мы можем всегда найти задачу (3), обладающую свойством фредгольмовости (которая однако не есть классическая BVP). Как вскоре будет видно, это утверждение следует из результатов Сили [13], [14], который, однако, не дошел до важного окончательного шага. Именно, для операторов $\widehat{D}$, для которых не существует классических BVP, он рассмотрел только задачу с однородными краевьми условиями $(g=0)$ и с псевдодифференциальным оператором $\widehat{B}$. Мы отметим также, что работы Сили существенно используют и развивают идеи Кальдерона ([12], см. также [16]), который первым ввел проекторы данного типа для изучения краевых задач. Близкие результаты получены также Буте де Монвелем [17] и Хёрмандером [18]. Проекторы Кальдерона нашли также свое применение в дифференциальных уравнениях и математической физике (см. [1], [18]-[22]). 
1.2. Теорема конечности (абстрактный случай). Покажем, как идеи Сили могут быть использованы в наших целях. Для прояснения основной идеи сначала приведем интуитивные соображения, а затем обсудим их более подробно. Итак, прежде всего отметим, что оператор $\widehat{D}$ может быть распространен до эллиптического псевдодифференциального оператора на дубле $2 M$ многообразия $M$ (заметим, что $2 M$ является замкнутым компактньм многообразием). Для любой функции $f \in H^{s-m}(M, F)$ определим непрерывный оператор продолжения на дубль $2 M$ с сохранением $(s-m)$-гладкости. Поскольку оператор $\widehat{D}$ является эллиптическим, то, как хорошо известно (с точностью до конечномерного дефекта, которым можно пренебречь во фредгольмовой теории), можно использовать правый почти обратный к оператору $\widehat{D}$ для того, чтобы редуцировать задачу (3) к задаче того же самого вида, однако уже с $f=0$ (и с некоторым сечением $g$, вообше говоря, отличным от первоначального):

$$
\left\{\begin{array}{l}
\widehat{D} u=0, \\
\widehat{B}\left(j_{X}^{m-1}(u)\right)=g, \quad u \in H^{s}(M, E) .
\end{array}\right.
$$

Через

$$
N(\widehat{D}, s)=\left\{u \in H^{s}(M, E): \widehat{D} u=0\right\}
$$

обозначим ядро оператора (2). Введем линейное многообразие

$$
R_{0}(\widehat{D}, s)=j_{X}^{m-1}(N(\widehat{D}, s)) \subset \mathscr{H}_{m}^{s-1 / 2}(X, E),
$$

т.е. пространство граничных данных для решений из $H^{s}(M, E)$ однородного уравнения. Если $R_{0}(\widehat{D}, s)$ является подпространством, т.е. замкнуто, то мы можем надеяться, что любой оператор $\widehat{B}$ такой, что (см. (4))

$$
\left.\widehat{B}\right|_{R_{0}(\widehat{D}, s)}: R_{0}(\widehat{D}, s) \rightarrow \mathscr{L},
$$

является изоморфизмом или, по крайней мере, фредгольмовым оператором и приводит к фредгольмовой $\mathrm{BVP}(3)$. В частности, если $\mathscr{L}=R_{0}(\widehat{D}, s)$ а $\widehat{B}$ - непрерывный проектор на $\mathscr{L}$, то мы получаем простейший пример BVP. Сформулируем теперь точное утверждение. Введем следуюшее обозначение:

$$
N_{0}(\widehat{D})=\left\{u \in C^{\infty}(M): \widehat{D} u=0, j_{X}^{m-1}(u)=0\right\} .
$$

Тогда $N_{0}(\widehat{D})$ является конечномерньм пространством.

Сили доказал следующее утверждение.

Teоpema 1 [13], [14]. Существует оператор

$$
\widehat{S}:\left(C^{\infty}(X, E)\right)^{m} \rightarrow C^{\infty}(M, E)
$$

maкой, что

i) для любого s оператор $\widehat{S}$ продолжсается до непрерьвного отображения

$$
\widehat{S}: \mathscr{H}_{m}^{s-1 / 2}(X, E) \rightarrow N(\widehat{D}, s) \subset H^{s}(M, E) ;
$$


ii) пространство $N(\widehat{D}, s)$ есть прямая сумма пространств $N_{0}(\widehat{D})$ и $\widehat{S}\left(\mathscr{H}_{m}^{s-1 / 2}(X, E)\right)$, m.e.

$$
N(\widehat{D}, s)=N_{0}(\widehat{D}) \oplus \widehat{S}\left(\mathscr{H}_{m}^{s-1 / 2}(X, E)\right)
$$

iii) onepamop

$$
\widehat{P}^{+}=j_{X}^{m-1} \circ \widehat{S}: \mathscr{H}_{m}^{s-1 / 2}(X, E) \rightarrow \mathscr{H}_{m}^{s-1 / 2}(X, E)
$$

является непрерывным проектором на $R_{0}(\widehat{D}, s)$; более того, $\widehat{P}^{+}$есть псевдодифференциальныци оператор, чей главный символ $\sigma\left(\widehat{P}^{+}\right)(x, \xi)$ осуществляет проекиию на пространство $L^{-}(x, \xi)$ начальных данных решений объкновенного дифференциального уравнения

$$
\sigma(\widehat{D})\left(x, 0, \xi,-i \frac{\partial}{\partial t}\right) \varphi(t)=0
$$

таких, что $\varphi(t) \rightarrow 0$ при $t \rightarrow+\infty$.

СледСтВИЕ 1. Справедливо следующее: $R_{0}(\widehat{D}, s)=\operatorname{Im} \widehat{P}^{+}$замкнуто.

Далее, для каждого $s$ Сили строит ограниченный оператор

$$
\widehat{C}: H^{s-m}(M, E) \rightarrow H^{s}(M, E)
$$

такой, что

$$
\widehat{D} \widehat{C} f=f
$$

для любого $f \in H^{s-m}(M, E)$, ортогонального ${ }^{1}$ к конечномерному пространству $N_{0}\left(\widehat{D}^{*}\right)$. Другими словами, оператор $\widehat{C}$ есть правьй обратньй к оператору $\widehat{D}$ с точностью до конечномерных операторов.

Теперь мы можем сфформулировать теорему, касающуюся общих BVP.

ТЕОРема 2. Общая краевая задача (3) (или, что то же самое, оператор (5)) является фредгольмовой, если и только если таковым является оператор ограничения (6):

$$
\left.\widehat{B}\right|_{\operatorname{Im} \widehat{P}^{+}}: \operatorname{Im} \widehat{P}^{+} \rightarrow \mathscr{L} .
$$

ДокАзАТЕльство. Сначала сведем наше утверждение к случаю, когда правая часть $f$ равна нулю.

ЛЕмма 1. Задача (3) является фредгольмовой одновременно с задачей

$$
\left\{\begin{array}{l}
\widehat{D} u=0, \\
\widehat{B}\left(j_{X}^{m-1}(u)\right)=g \in \mathscr{L} .
\end{array}\right.
$$

Другими словами, оператор (5) является фредгольмовым, если и только если таковым является оператор

$$
\widehat{B} \circ j_{X}^{m-1}: N(\widehat{D}, s) \rightarrow \mathscr{L} .
$$

\footnotetext{
${ }^{1}$ Предполагается, что на $M$ выбрана некоторая мера, а также выбрана и зафикисирована некоторая эрмитова метрика в рассматриваемом расслоении.
} 
ДоКАЗАТЕЛЬСТво ЛЕммы 1. Очевидно, ядра операторов (5) и (12) совпадают. Изучим коядра. Утверждается, что коядро оператора (5) изоморфно коядру оператора (12) плюс (прямая сумма) $N_{0}\left(\widehat{D}^{*}\right)$. Действительно, пусть $f$ ортогонально к $N_{0}\left(\widehat{D}^{*}\right)$. Тогда в силу $(10)$ подстановка

$$
u=\widehat{C} f+\widetilde{u}
$$

сводит задачу (3) к задаче (11) для $\widetilde{u}$ и $g$, замененной на

$$
\widetilde{g}=g-\widehat{B} j_{X}^{m-1} \widehat{C} f .
$$

Поскольку $\operatorname{dim} N_{0}\left(\widehat{D}^{*}\right)<\infty$, то отсюда легко следует требуемое утверждение.

Теперь мы имеем разложение

$$
\widehat{B} \circ j_{X}^{m-1}: N(\widehat{D}, s) \stackrel{j_{X}^{m-1}}{\longrightarrow} R_{0}(\widehat{D}, s)=\operatorname{Im} \widehat{P}^{+} \stackrel{\widehat{B}}{\longrightarrow} \mathscr{L},
$$

и утверждение теоремы 2 может быть немедленно получено из леммы 1 и следующего утверждения.

Лemma 2. Onepamop

$$
j_{X}^{m-1}: N(\widehat{D}, s) \rightarrow R_{0}(\widehat{D}, s)
$$

является фредгольмовым.

ДокАЗАТЕльСТво ЛЕммы 2. По определению пространства $R_{0}(\widehat{D}, s)$ оператор (13) является эпиморфизмом. Теперь в силу (7) ядро оператора (13) есть в точности $N_{0}(\widehat{D})$, которое конечномерно. Это завершает доказательство леммы 2 .

1.3. Теорема конечности (псевдодифференциальный случай). В применении часто бывает важным описать пространство $\mathscr{L}$ и краевой оператор $\widehat{B}$ в явных терминах. Вид “простейшей” фредгольмовой BVP, в которой $\widehat{B}=\widehat{P}^{+}$и $\mathscr{L}=\operatorname{Im} \widehat{P}^{+}$, дает следуюшее описание: $\widehat{B}$ должен быть псевдодифференциальным оператором, действующим в сечении векторных расслоений над $X$,

$$
\widehat{B}: \mathscr{H}_{m}^{s-1 / 2}(X, E) \rightarrow H^{\sigma}(X, G),
$$

а подпространство $\mathscr{L} \subset H^{\sigma}(X, G)$ должно быть описано как образ некоторого псевдодифференциального оператора

$$
\widehat{P}: H^{\sigma}(X, G) \rightarrow H^{\sigma}(X, G)
$$

(для простоты предполагается, что оператор $\widehat{P}$ является псевдодифференциальньм оператором порядка нуль). Более того, предполагается также, что главньй символ $\sigma(\widehat{P})(x, \xi)$ является оператором проектирования в $\left(\pi^{*} G\right)_{(x, \xi)},(x, \xi) \in T_{0}^{*} X$, где

$$
\pi: T_{0}^{*} X \rightarrow X
$$

- естественная проекция, образ оператора $\widehat{P}$ замкнут и $\operatorname{Im} \widehat{B} \subset \operatorname{Im} \widehat{P}$. Образ $\operatorname{Im} \widehat{P}$ наделим структурой гильбертова пространства, индуцированной из $H^{\sigma}(X, G)$.

Рассмотрим обшую краевую задачу для эллиптического оператора $\widehat{D}$

$$
\left\{\begin{array}{l}
\widehat{D} u=f \in H^{s-m}(M, F), \\
\widehat{B}\left(j_{X}^{m-1} u\right)=g \in \operatorname{Im} \widehat{P} \subset H^{\sigma}(X, G)
\end{array}\right.
$$

с неизвестной функцией $u \in H^{s}(M, E)$. 
Теорема 3. Предположим, что выполнено следующее условие.

(GSL) Для любой точки $(x, \xi) \in T_{0}^{*} X$ главный символ $\sigma(\widehat{B})(x, \xi)$ оператора $\widehat{B}$ индуцирует изоморфизм между пространствами ${ }^{2} L^{-}(x, \xi)$ и $\operatorname{Im} \sigma(\widehat{P})(x, \xi)$.

Тогда задача (14) является фредгольмовой. Другими словами, оператор

$$
\left(\widehat{D}, \widehat{B} \circ j_{X}^{m-1}\right): H^{s}(M, E) \rightarrow H^{s-m}(M, F) \oplus \operatorname{Im} \widehat{P}
$$

является фредгольмовым оператором.

Условие (GSL) назовем условием коэриитивности или обобщенныцм условием Шапиро-Лопатинского. В случае $\widehat{P}=1$ приходим к обычному условию Шапиро-Лопатинского (см., например, [1]). Это будет обсуждено в п. 1.4. Преимушество общего краевого условия состоит в том, что краевая задача, удовлетворяюшая этому условию, может быть поставлена для произвольного эллиптического оператора $\widehat{D}$ (достаточно взять ${ }^{3} \widehat{B}=\widehat{P}=\widehat{P}^{+}$).

ДоКАЗАТЕЛЬСТво ТЕОРЕМЫ 3 . В силу теоремы 2 достаточно доказать, что оператор

$$
\widehat{B}: \operatorname{Im} \widehat{P}^{+} \rightarrow \operatorname{Im} \widehat{P}
$$

является фредгольмовым оператором. Сделаем сначала техническое замечание. Оператор $\widehat{P}^{+}$действует в пространстве

$$
\mathscr{H}_{m}^{s-1 / 2}(X, E)=\bigoplus_{j=0}^{m-1} H^{s-1 / 2-j}(X, E)
$$

и имеет в этом пространстве порядок нуль; поэтому порядки матрищ, входящих в оператор $\widehat{P}^{+}$, меняются в соответствии с порядками прямых слагаемых в разложении $(17)$, и главный символ оператора $\widehat{P}^{+}$, о котором мы говорили выше, должен быть определен как главный символ в смысле Дуглиса-Ниренберга [23]. Разумеется, более удобно было бы иметь дело с обычньми (однородными) главными символами. Чтобы этого достичь, возьмем обратимый эллиптический псевдодифференциальньй оператор первого порядка $\widehat{\Lambda}_{\text {в }} C^{\infty}(X, E)$ и используем изоморфизм

$$
\widehat{U}=\operatorname{diag}\left(\widehat{\Lambda}^{m-1}, \widehat{\Lambda}^{m-2}, \ldots, \widehat{\Lambda}^{0}\right): \mathscr{H}_{m}^{s-1 / 2}(X, E) \rightarrow\left(H^{s-m+1 / 2}(X, E)\right)^{m} .
$$

Таким образом, мы заменим $\widehat{P}^{+}$на $\widehat{U} \widehat{P}^{+} \widehat{U}^{-1}$ и $\widehat{B}$ на $\widehat{B} \widehat{U}^{-1}$ и обозначим новые полученные операторы теми же самыми буквами.

Теперь оператор $\widehat{P}^{+}$имеет порядок 0 , оператор $\widehat{B}$ имеет порядок $s-m+\frac{1}{2}-\sigma$, а главный символ $\sigma(\widehat{B})$ оператора $\widehat{B}$ осушествляет изоморфизм образов символов $\sigma\left(\widehat{P}^{+}\right)$и $\sigma(\widehat{P})$. Ниже мы будем иногда писать $A$ вместо $\sigma(\widehat{A})$ для главного символа любого псевдодифференциального оператора $\widehat{A}$.

\footnotetext{
${ }^{2}$ Напомним, что пространство $L^{-}(x, \xi)$ строится с помощью главного символа оператора $\widehat{D}$ как пространство решений уравнения (9), убывающих при $t \rightarrow+\infty$.

${ }^{3}$ Более точно, необходимо редуцировать порядок, поскольку оператор $\widehat{P}$ является оператором нулевого порядка.
} 
Поскольку любая короткая последовательность векторных расслоений расщепляется, то легко найти символы

$$
R_{1}, R_{2} \in \operatorname{Hom}\left(\pi^{*} G, \pi^{*} E^{m}\right), \quad \pi: T_{0}^{*} X \rightarrow X,
$$

однородные порядка $\sigma+m-s-\frac{1}{2}$, такие, что

$$
\begin{gathered}
P^{+} R_{i}=R_{i}, \quad i=1,2, \\
R_{1} B=P^{+}, \quad B R_{2}=P .
\end{gathered}
$$

Пусть

$$
\widehat{\mathscr{R}}_{i}=\widehat{P}^{+} \widehat{R}_{i}, \quad i=1,2
$$

Тогда

$$
\widehat{\mathscr{R}}_{i}(\operatorname{Im} \widehat{P}) \subset \operatorname{Im} \widehat{P}^{+}
$$

И

$$
\begin{gathered}
\widehat{\mathscr{R}}_{1} \widehat{B}=\widehat{P}^{+}+\widehat{Q}_{1}, \\
\widehat{B} \widehat{\mathscr{R}}_{2}=\widehat{P}+\widehat{Q}_{2},
\end{gathered}
$$

где $\widehat{Q}_{i}, i=1,2,-$ псевдодифференциальные операторы порядка -1 на $X$ (и, следовательно, компактные операторы) и, более того,

$$
\begin{gathered}
\widehat{Q}_{1} \operatorname{Im}\left(\widehat{P}^{+}\right) \subset \operatorname{Im}\left(\widehat{P}^{+}\right), \\
\widehat{Q}_{2} \operatorname{Im}(\widehat{P}) \subset \operatorname{Im}(\widehat{P})
\end{gathered}
$$

(последнее включение имеет место в силу того, что $\operatorname{Im} \widehat{B} \subset \operatorname{Im} \widehat{P}$ ). Теперь, ограничивая (18) и (19) на $\operatorname{Im} \widehat{P}^{+}$и $\operatorname{Im} \widehat{P}$, соответственно, получаем

$$
\begin{gathered}
\widehat{\mathscr{R}}_{1} \widehat{B}=1_{\operatorname{Im} \widehat{P}^{+}}+\widehat{Q}_{1}, \\
\widehat{B} \widehat{\mathscr{R}}_{2}=1_{\operatorname{Im} \widehat{P}}+\widehat{Q}_{2}+\left.(\widehat{P}-1)\right|_{\operatorname{Im} \widehat{P}} .
\end{gathered}
$$

ЛЕмма 3. Оператор $\left.(\widehat{P}-1)\right|_{\operatorname{Im} \widehat{P}}$ является компактньцм.

ДокАЗАтЕльство леммы 3 . Пусть $S$ - единичная сфера в пространстве $\operatorname{Im} \widehat{P}$. Рассмотрим ограниченньй оператор

$$
\widetilde{P}: H^{\sigma}(X, G) / \operatorname{Ker} \widehat{P} \rightarrow \operatorname{Im} \widehat{P}
$$

индуцированный оператором $\widehat{P}$. Этот оператор является взаимно однозначным, и поэтому пространство Im $\widehat{P}$ замкнуто. Из теоремы Банаха следует, что оператор $\widetilde{P}^{-1}$ ограничен и, таким образом, $\widetilde{P}^{-1}(S)$ является ограниченным множеством в $H^{\sigma}(X, G) / \operatorname{Ker} \widehat{P}$. Следовательно, существует такое ограниченное множество $\widetilde{S} \subset$ $H^{\sigma}(X, G)$, что $S=\widehat{P}(\widetilde{S})$. Теперь

$$
(\widehat{P}-1)(S)=\left(\widehat{P}^{2}-\widehat{P}\right)(\widetilde{S})
$$


- относительно компактное подмножество в пространстве $H^{\sigma}(X, G)$, поскольку $\widehat{P}^{2}-\widehat{P}$ является оператором порядка -1 (напомним, что $P^{2}=P$ ). Лемма 3 доказана.

Из (20) следует, что $\widehat{\mathscr{R}}_{1}$ и $\widehat{\mathscr{R}}_{2}$ являются, соответственно, левым и правьм регуляризаторами оператора $\widehat{B}$ (см. (16)). Таким образом, оператор (16) является фредгольмовьм, что завершает доказательство теоремы 3.

Доказательство теоремы 3 может быть также дано с помощью явного построения почти обратного оператора к оператору (15). Это построение может быть осушествлено в совершенно "классическом" духе: замораживание коэффициентов в произвольной точке края, преобразование Фурье по касательным переменнњм, рассмотрение семейства обыкновенных дифференциальных уравнений и т. д. Читатель может найти полное доказательство в работе [15].

1.4. Условие Шапиро-Лопатинского. Если $\widehat{P}$ - тождественный оператор, $\widehat{P}=1$, тогда задача (14) превращается в классическую краевую задачу для эллиптического оператора $\widehat{D}$ с краевыми условиями, определяемьми оператором $\widehat{B}$ (правая часть $g$ в краевых условиях предполагается лежащей в образе всего пространства Соболева $\left.H^{\sigma}(X, G)\right)$. Заметим, что главный символ $P(x, \xi)$ оператоpa $\widehat{P}$

$$
P(x, \xi):\left(\pi^{*} G\right)_{(x, \xi)} \equiv G_{x} \rightarrow\left(\pi^{*} G\right)_{(x, \xi)},
$$

в этом случае является тождественным оператором и, следовательно,

$$
\operatorname{Im} P(x, \xi)=\left(\pi^{*} G\right)_{(x, \xi)}
$$

Поэтому условие (GSL) означает требование, чтобы символ оператора $B$ определял изоморфизм

$$
B: L^{-} \rightarrow \pi^{*} G
$$

расслоений над многообразием $X$, где, разумеется,

$$
L^{-} \rightarrow T_{0}^{*} X
$$

есть расслоение со слоем $L^{-}(x, \xi)$ в любой точке $(x, \xi) \in T_{0}^{*} X$ и $\pi^{*}: T_{0}^{*} M \rightarrow M-$ естественная проекция.

Таким образом, это условие является обычным условием Шапиро-Лопатинского.

Мы видим, что классические краевые задачи, удовлетворяющие условию ШІапиро-Лопатинского, существуют, если и только если расслоение $L^{-}$изоморфно обратному образу при естественной проекиии некоторого расслоения над $X$.

Препятствие к существованию такого изоморфизма может быть описано следуюшим образом (см. [3], [4]). Заметим прежде всего, что достаточно рассматривать косферическое расслоение $S^{*} X$ вместо $T_{0}^{*} X$. Для каждой точки $(x, \xi) \in S^{*} X$ рассмотрим обыкновенный дифференциальный оператор

$$
\widehat{D}(x, \xi) \equiv \sigma(\widehat{D})\left(x, 0, \xi,-i \frac{\partial}{\partial t}\right): H^{m}\left(\mathbb{R}_{+}\right) \rightarrow L_{2}\left(\mathbb{R}_{+}\right)
$$


с постоянными коэффициентами (см. (9)). Поскольку коэффициент при $(\partial / \partial t)^{m}$ не равен нулю, отсюда следует, что $\{\widehat{D}(x, \xi)\}$ определяет семейство фредгольмовых операторов, параметризованных точками $(x, \xi) \in S^{*} X$, и следовательно, корректно определен $K$-теоретический индекс [24]

$$
\operatorname{index}\{\widehat{D}(x, \xi)\} \in K\left(S^{*} X\right) .
$$

Заметим, что

$$
\operatorname{index}\{\widehat{D}(x, \xi)\}=\left[L^{-}\right],
$$

где $\left[L^{-}\right]-$класс расслоения $\left.L^{-}\right|_{S^{*} X}$ в группе $K\left(S^{*} X\right)$, поскольку имеет место изоморфизм

$$
\operatorname{Ker} \widehat{D}(x, \xi)=L^{-}(x, \xi)
$$

И

$$
\text { Coker } \widehat{D}(x, \xi)=\{0\}
$$

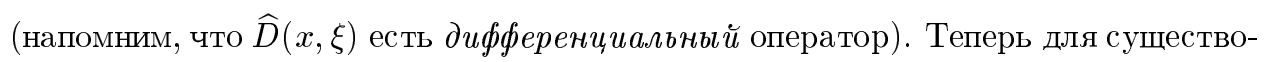
вания изоморфизма (21) необходимо, чтобы

$$
\operatorname{index}\{\widehat{D}(x, \xi)\} \in \pi^{*} K(X),
$$

где $\pi: S^{*} X \rightarrow X-$ каноническая проекция.

\section{§2. Примеры}

2.1. Оператор Коши-Римана. Рассмотрим оператор $\partial / \partial \bar{z}$ на комплексном многообразии $M$ (комплексной) размерности 1. Очевидно, этот оператор является эллиптическим. Предположим, что граница $X$ многообразия $M$ является чисто вешественной, т.е. существуют координаты в окрестности края такие, что уравнение $z=x+i y$ многообразия $X$ есть $\{y=0\}$. В этих координатах мы имеем

$$
\widehat{D}=\frac{\partial}{\partial \bar{z}}=\frac{1}{2}\left(\frac{\partial}{\partial x}+i \frac{\partial}{\partial y}\right)=\frac{i}{2}\left(\frac{\partial}{\partial y}-i \frac{\partial}{\partial x}\right) .
$$

Символ этого оператора есть

$$
\widehat{D}(p, \xi)=\frac{i}{2}(p+\xi)
$$

(переменная $y$ играет роль координаты $t$ в обших построениях). В качестве расслоения $E$ возьмем одномерное тривиальное расслоение над $X$. Для каждой точки $x_{0} \in X$ имеем, очевидно, $p_{1}(\xi)=-\xi$. Далее,

$$
\begin{array}{ll}
L^{+}(\xi)=\mathbb{R}, \quad L^{-}(\xi)=\{0\}, \quad \xi<0, \\
L^{-}(\xi)=\mathbb{R}, \quad L^{+}(\xi)=\{0\}, \quad \xi>0 .
\end{array}
$$

Следовательно,

$$
P^{+}(\xi)=\theta(-\xi), \quad P^{-}(\xi)=\theta(\xi)
$$


где $\theta$ - функция Хевисайда.

Поскольку размерность пространства $L^{-}(\xi)$ различна для $\xi<0$ и $\xi>0$, отсюда следует, что $L^{-}(\xi)$ неизоморфна обратному образу никакого расслоения над $X$.

Пусть теперь

$$
\left\{\begin{array}{l}
\widehat{D} u=f \\
\left.\widehat{B} u\right|_{y=0}=g
\end{array}\right.
$$

- краевая задача для оператора $\widehat{D}$. Очевидно, расслоение $F$ (см. п.1.1), используемое в граничных условиях, должно быть одномерным, если мы хотим удовлетворить условию Шапиро-Лопатинского. Функция $B(x, \xi)$ в нашем случае - скалярная. Обобшенное условие Шапиро-Лопатинского дает изоморфизмы

$$
\begin{aligned}
& B(x, \xi): \mathbb{R} \rightarrow \mathbb{R}, \quad \xi>0, \\
& B(x, \xi):\{0\} \rightarrow \mathbb{R}, \quad \xi<0,
\end{aligned}
$$

на некоторых подпространствах. Ясно, что условие удовлетворяется, если $B(x, \xi) \neq 0$ для $\xi>0, B(x, \xi)=0$ для $\xi<0$, и проектор $P(x, \xi)$ совпадает с $P^{-}(\xi)$. При этих условиях задача $(22) \mathrm{c}$

$$
u \in H^{s}(M), \quad f \in H^{s-1}(M), \quad g \in \operatorname{Im} \widehat{P}^{-}(\xi) \subset H^{s-1 / 2}(M)
$$

является фредгольмовой. Отметим, что образ оператора $\widehat{P}^{-}(\xi)$ называется $n р о-$ странством Харди [10], [11].

2.2. Оператор Эйлера. Рассмотрим четномерное риманово многообразие $M$ с краем $\partial M=X$. Предполагается, что вблизи края метрика является прямьм произведением метрики на $X$ и стандартной метрики $d t^{2}$ на $\mathbb{R}^{1}$. Пусть

$$
d+\delta: \Lambda^{\mathrm{ev}}(M) \rightarrow \Lambda^{\text {odd }}(M)
$$

- оператор Эйлера (см. [25]-[28]) на $M$. В координатах $(t, x)$ вблизи $X$ этот оператор может быть переписан в виде

$$
\left(\begin{array}{cc}
\frac{\partial}{\partial t} & \left(d_{X}+\delta_{X}\right)_{\text {odd }} \\
\left(d_{X}+\delta_{X}\right)_{\mathrm{ev}} & \frac{\partial}{\partial t}
\end{array}\right):\left(\begin{array}{c}
\Lambda^{\mathrm{ev}}(X) \\
\Lambda^{\text {odd }}(X)
\end{array}\right) \rightarrow\left(\begin{array}{c}
\Lambda^{\mathrm{ev}}(X) \\
\Lambda^{\text {odd }}(X)
\end{array}\right)
$$

где $d_{X}$ и $\delta_{X}$ - соответственно, внешний дифференциал на $X$ и его метрический сопряженный. Для вычисления символа оператора Эйлера заметим, что

i) символ оператора $d_{X}$ есть внешнее умножение на $i \xi d x$;

ii) символ оператора $\delta_{X}$ есть внутреннее умножение на $-i V_{\xi}$, где $V_{\xi}$ - вектор, соответствующий $\xi d x$ относительно метрики $g_{X}$;

iii) имеет место соотношение

$$
\left.\left.\left.\left.\left(\xi d x \wedge-V_{\xi}\right\rfloor\right)^{2} \omega=-\left((\xi d x \wedge)\left(V_{\xi}\right\rfloor\right)+\left(V_{\xi}\right\rfloor\right)(\xi d x\rfloor\right)\right) \omega=-\xi^{2} \omega,
$$

где $\wedge$ и 」- операторы внешнего и внутреннего умножения, соответственно [29]. 
Символ оператора (23) имеет вид:

$$
D(p, \xi)=\left(\begin{array}{cc}
p & \left.i\left(\xi d x \wedge-V_{\xi}\right\rfloor\right) \\
\left.i\left(\xi d x \wedge-V_{\xi}\right\rfloor\right) & p
\end{array}\right) .
$$

Найдем спектральные точки и соответствующие подпространства операторного семейства.

Характеристическое уравнение имеет вид:

$$
\left(\begin{array}{cc}
p & \left.i\left(\xi d x \wedge-V_{\xi}\right\rfloor\right) \\
\left.i\left(\xi d x \wedge-V_{\xi}\right\rfloor\right) & p
\end{array}\right)\left(\begin{array}{c}
\omega^{\mathrm{ev}} \\
\omega^{\text {odd }}
\end{array}\right)=0
$$

где (заметим, что, как это следует из общих рассмотрений, $p \neq 0$ для $\xi \neq 0$ )

$$
\left.p \omega^{\mathrm{ev}}+i\left(\xi d x \wedge-V_{\xi}\right\rfloor\right) \omega^{\text {odd }}=0,
$$

T.e.

$$
\left.\omega^{\mathrm{ev}}=-\frac{i}{p}\left(\xi d x \wedge-V_{\xi}\right\rfloor\right) \omega^{\mathrm{odd}}
$$

и

$\left.\left.0=i\left(\xi d x \wedge-V_{\xi}\right\rfloor\right) \omega^{\mathrm{ev}}+p \omega^{\text {odd }}=\frac{1}{p}\left(\xi d x \wedge-V_{\xi}\right\rfloor\right)^{2} \omega^{\text {odd }}+p \omega^{\text {odd }}=\frac{1}{p}\left(p^{2}-|\xi|^{2}\right) \omega^{\text {odd }}$.

Отсюда имеем

$$
p(\xi)=p_{ \pm}(\xi)= \pm|\xi|
$$

Следовательно, если $p= \pm|\xi|$,

$$
\left.\omega^{\mathrm{ev}}=\mp \frac{i}{|\xi|}\left(\xi d x \wedge-V_{\xi}\right\rfloor\right) \omega^{\mathrm{odd}}
$$

и $\omega^{\text {odd }} \in \Lambda^{\text {odd }}(X)$ - произвольная форма, то уравнение (24) выполняется. Поэтому спектр $D(p, \xi)$ состоит из двух точек $p= \pm|\xi|$ для $\xi \neq 0$ и соответствуюшее собственное подпространство описывается соотношением $(25)$, где $\omega^{\text {odd }}$ пробегает все пространство $\Lambda^{\text {odd }}(X)$. Аналогично мы можем доказать, что собственное пространство описывается формулой

$$
\left.\omega^{\text {odd }}=\mp \frac{i}{|\xi|}\left(\xi d x \wedge-V_{\xi}\right\rfloor\right) \omega^{\mathrm{ev}},
$$

где $\omega^{\mathrm{ev}}$ пробегает пространство $\Lambda^{\mathrm{ev}}(X)$.

Уравнения $(25)$ и $(26)$ показьвают, что проекторы

$$
P_{\mathrm{ev}}: \Lambda(X)=\Lambda^{\mathrm{ev}}(X) \oplus \Lambda^{\mathrm{odd}}(X) \rightarrow \Lambda^{\mathrm{ev}}(X)
$$

и

$$
P_{\text {odd }}: \Lambda(X)=\Lambda^{\text {ev }}(X) \oplus \Lambda^{\text {odd }}(X) \rightarrow \Lambda^{\text {odd }}(X)
$$


являются изоморфизмами пространства $L^{-}(\xi)$, отвечающего собственному значению $p_{-}(\xi)=-|\xi|$ на $\Lambda^{\mathrm{ev}}(X)$ и $\Lambda^{\text {odd }}(X)$, соответственно. Следовательно, для оператора Эйлера мы имеем классические, т.е. удовлетворяющие условию Шапиро-Лопатинского, фредгольмовы краевые задачи

$$
\left\{\begin{array} { l } 
{ \widehat { D } u = f , } \\
{ P _ { \text { ev } } u | _ { X } = g _ { 1 } }
\end{array} \quad \text { и } \quad \left\{\begin{array}{l}
\widehat{D} u=f, \\
\left.P_{\text {odd }} u\right|_{X}=g_{2},
\end{array}\right.\right.
$$

где

$$
\widehat{D}=d+\delta: H^{s}\left(M, \Lambda^{\mathrm{ev}}(M)\right) \rightarrow H^{s-1}\left(M, \Lambda^{\text {odd }}(M)\right)
$$

- оператор Эйлера и элементы $g_{1}$ и $g_{2}$ принадлежат пространствам

$$
H^{s-1 / 2}\left(X, \Lambda^{\mathrm{ev}}(X)\right), \quad H^{s-1 / 2}\left(X, \Lambda^{\text {odd }}(X)\right),
$$

соответственно.

\section{Список литературы}

1. Agranovich M. Elliptic boundary problems // Encyclopaedia of Mathematical Sciences / ed. M. S. Agranovich, Yu. V. Egorov, M. A. Shubin. Berlin: Springer-Verlag, 1997. P. 1-144.

2. Hörmander L. The Analysis of Linear Partial Differential Operators. II. Berlin: SpringerVerlag, 1983.

3. Atiyah M.F., Bott R. The index problem for manifolds with boundary // Bombay Colloquium on Differential Analysis. Oxford: Oxford Univ. Press, 1964. P. 175-186.

4. Rempel S., Schulze B.-W. Index Theory of Elliptic Boundary Problems. Berlin: AkademieVerlag, 1982.

5. Schulze B.-W. Boundary value problems and edge pseudo-differential operators // Microlocal Analysis and Spectral Theory / ed. Luigi Rodino. Dordrecht: Kluwer Acad. Publ., 1997. P. 165-226.

6. Boutet de Monvel L.. Boundary problems for pseudodifferential operators // Acta Math. 1971. V. 126. P. 11-51.

7. Atiyah M., Patodi V., Singer I. Spectral asymmetry and Riemannian geometry I, II, III // Math. Proc. Cambridge Philos. Soc. 1975. V. 77. P. 43-69; 1976. V. 78. P. 405-432; 1976. V. 79. P. 315-330.

8. Понтрягин Л. С. Характеристические циклы дифференцируемых многообразий // Матем. сб. 1947. Т. 21. С. 233-284.

9. Бицадзе A.B. Краевые задачи для эллиптических уравнений второго порядка. М.: Наука, 1966.

10. Привалов И. И. Граничные свойства аналитических функций. М.-Л.: ГИТТЛ, 1950.

11. Brenner V.A., Shargorodsky E.M. Boundary value problems for elliptic pseudodifferential operators // Encyclopaedia of Mathematical Sciences / ed. M.S. Agranovich, Yu. V. Egorov, M. A. Shubin. Berlin: Springer-Verlag, 1997. P. 145-215.

12. Calderón A.P. Boundary value problems for elliptic equations // Outlines of the Joint Soviet-American Symposium on Partial Differential Equations. Novosibirsk: Novosibirsk Univ., 1963. P. 303-304.

13. Seeley R. T. Singular integrals and boundary value problems // Amer. J. Math. 1966. V. 88. P. 781-809.

14. Seeley R. T. Topics in pseudo-differential operators // Pseudo-Differential Operators / ed. L. Nirenberg. Roma. C.I.M.E. Conference on pseudo-differential operators, Stresa 1968, Roma: Cremonese, 1969. P. 167-305.

15. Schulze B.-W., Sternin B., Shatalov V. On General Boundary Value Problems for Elliptic Equations // Preprint № 97/35. Univ. Potsdam, Institut für Mathematik, Potsdam, November 1997. 
16. Calderón A.P. Lecture Notes on Pseudo-Differential Operators and Elliptic Boundary Value Problems, I. Buenos Aires: Instituto Argentino de Mathematica, 1976.

17. Boutet de Monvel L. Comportement d'un opérateur pseudo-différentiel sur une variété à bord, I, II // J. Anal. Math. 1966. V. 17. P. 241-253; 255-304.

18. Hörmander L. Pseudo-differential operators and non-elliptic boundary problems // Ann. of Math. (2) 1966. V. 83. P. 129-209.

19. Grubb G. Boundary value problems for systems of partial differential operators of mixed order // J. Funct. Anal. 1977. V. 26. № 2. P. 131-165.

20. Grubb G., Seeley R. Weakly parametric pseudo-differential operators and Atiyah-PatodiSinger boundary problems // Invent. Math. 1995. V. 121. P. 481-529.

21. Hörmander L. The Analysis of Linear Partial Differential Operators. III. Berlin: SpringerVerlag, 1985.

22. Palais R. S. Seminar on the Atiyah-Singer Index Theorem. Princeton, NJ: Princeton Univ. Press, 1965.

23. Douglis A., Nirenberg L. Interior estimates for elliptic systems of partial differential equaions // Comm. Pure Appl. Math. 1955. V. 8. P. 503-538.

24. Atiyah M.F. K-Theory. The Advanced Book Program. Cambridge: Addison-Wesley, 1989.

25. Atiyah M.F., Singer I. M. The index of elliptic operators III // Ann. of Math.(2) 1968. V. 87. P. $546-604$.

26. Gilkey P. B. The boundary integrand in the formula for the signature and Euler characteristic of Riemanian manifold with boundary // Adv. Math. 1975. V. 15. P. 334-360.

27. Дезин A. А. Инвариантные дифференциальные операторы и граничные задачи // Труды МИАН. 1962. Т. 68.

28. Дезин А. А. Многомерньй анализ и дискретные модели. М.: Наука, 1990.

29. Sternberg S. Lectures on Differential Geometry. Englewood Cliffs, NJ: Prentice-Hall, 1964.

МГУ им. М.В. Ломоносова;

МГУ им. М.В. Ломоносова;

Поступила в редакцию

Потсдамский университет

14.05 .1998 